\title{
Dyke-Davidoff-Masson Syndrome as a rare congenital hemiatrophy: a case report
}

Azubuike Benjamin Nwako ${ }^{*}$, Charles Emeka Nwolisa², Okechukwu Francis Nwako ${ }^{3}$, Magaret-Lorritta Chidimma Nwako ${ }^{4}$

${ }^{1}$ Department of Paediatrics, Queen Elizabeth II Hospital Maseru, Maseru, Lesotho

${ }^{2}$ Department of Paediatrics, Federal Medical Centre Owerri, Owerri, Imo State, Nigeria

${ }^{3}$ Department of Internal Medicine, Federal Medical Centre Owerri, Owerri, Imo State, Nigeria

${ }^{4}$ Independent Researcher, Hillsview, Maseru, Lesotho

\begin{abstract}
Introduction: Dyke-Davidoff-Masson syndrome (DDMS) is a rare condition in childhood with very few cases reported in sub-Saharan Africa. Typically, the patient presents with facial asymmetry, seizures and hemiparesis. Radiological findings include cerebral hemiatrophy, ipsilateral lateral ventricular dilatation and hypertrophy of the calvarium and sinuses.

Case presentation: We present the report of a 3-year-old male with weakness of right upper and lower limbs, facial asymmetry and seizures. Physical examination showed a well-nourished child with squint of the right eye. There were brisk tendon reflexes with right-sided hemiplegia which is spastic with left limb preference. Computer tomography of the brain showed atrophy of the left cerebral hemisphere, ipsilateral lateral ventricular dilatation and thickening of the ipsilateral cranium. The diagnosis of DykeDavidoff-Masson syndrome was made. He was treated with sodium valproate and lamotrigine and presently physiotherapy and he had remained seizure free.

Conclusion: Dyke-Davidoff-Masson syndrome still affects children despite being a rare condition. There is need for appropriate clinical and radiological assessment for diagnosis of DDMS. Early identification and appropriate treatment will improve the general outcome of children with DDMS.
\end{abstract}

Keywords: Dyke-Davidoff-Masson syndrome, congenital cerebral hemiatrophy, seizures

\section{Introduction}

Dyke-Davidoff-Masson syndrome (DDMS) is a disorder first identified in 1933 by Dyke, Davidoff and Masson in nine patients with hemiplegia and cranial asymmetry using cranial X-ray findings. Few cases have been reported in the sub-Sahara Africa including Nigeria and Ethiopia (Uduma et al., 2013). It occurs in both gender but with male preponderance (73.5\%) (Duncan et al, 2014). The condition is characterized by unequal size of the brain due to one-sided cerebral atrophy, seizures, mental retardation, hemiplegia, frontal sinus and skull abnormalities (Duncan et al, 2014). It may be congenital from congenital malformation and vascular abnormalities or acquired in the intra- and post-partum period from infection and birth trauma (Sharma et al., 2006). Cerebral malaria has been implicated as a cause of DDMS due to suspected cerebral occlusion (Karuppiah et al., 2009). The patients more often present with seizure disorders, facial asymmetry, hemiparesis and mental retardation (Dutta et al., 2016). Computer tomography (CT) scan may demonstrate unilateral cerebral atrophy, increased sulci, ipsilateral ventricular dilatation, skull thickening, elevation of petrous sinuses and midline shift on the ipsilateral side of the atrophy (Piro et al., 2013). DDMS has also been known to present as hemiplegic cerebral palsy with focal seizure (Sharawat et al, 2019).

The prognosis depends on the presence and severity of seizure disorders. There are other conditions which may present with cerebral hemiatrophy such as Herpes Simplex Encephalitis, StugeWeber syndrome, Fishman syndrome and Rasmussen encephalitis (Uduma et al., 2013). We present

* Correspondence: Dr Azubuike Benjamin Nwako (drabnwakomba@gmail.com) 
the report of a 3-year-old male with clinical features and computer tomography findings in keeping with Dyke-Davidoff-Masson syndrome. This is the first reported case of DDMS in Lesotho to the best of our knowledge.

\section{Case Presentation}

Our patient was a three-year-old male who presented initially at the age of one year to the paediatric clinic of the Queen Elizabeth II Hospital Maseru Lesotho. His presenting complaints were weakness of right upper and lower limbs, right facial asymmetry and seizures all noticed at about one year of age. The mother observed left hand preference with infrequent and slow use of the right hand.

Seizures was said to have developed about a month after the onset of right-sided weakness and was generalized tonic-clonic seizures. It was observed about once a month and each episode lasted between two to three minutes in duration. The seizure was not associated with fever. There was no history of trauma immediately preceding the onset of the symptoms.

The pregnancy was apparently unremarkable and the mother had no illnesses reported during pregnancy. The mother had normal vaginal delivery of a preterm low birth weight with birth weight of 2400 grams. The child was said to have cried immediately after birth. He was however managed postpartum for hypothermia and hypoglycemia from suspected neonatal sepsis and was discharged after six days on admission. On discharge, he was clinically stable and the primitive reflexes were intact and symmetrical. There was no evidence of any neurological deficit. There was no neonatal jaundice or neonatal seizure observed.

His developmental milestone was slightly delayed; sitting at ten months, walking at two years and made first word at two years. He currently can make two-word sentence. There was differential movement of the child towards the right while walking. He was in preschool.

On general examination, he was well-nourished with weight-for-height Z-score between median and one standard-deviation, has right-sided facial asymmetry and hemiplegic gait. The head circumference was $51 \mathrm{~cm}$ which was within normal range. There was no cranial nerve seven palsy but the child has squint on the right eye. Other cranial nerves were generally intact. There were brisk deep tendon reflexes with right-sided hemiplegia which was spastic with left limb preference. The deep tendon reflexes on the left side were normal. The power in the right upper and lower limbs were reduced but normal on the left side. Computer tomography scan showed atrophy of the left cerebral hemisphere with ipsilateral lateral ventricular dilatation (Figures 1 and 2). There was also thickening of the ipsilateral cranium. 


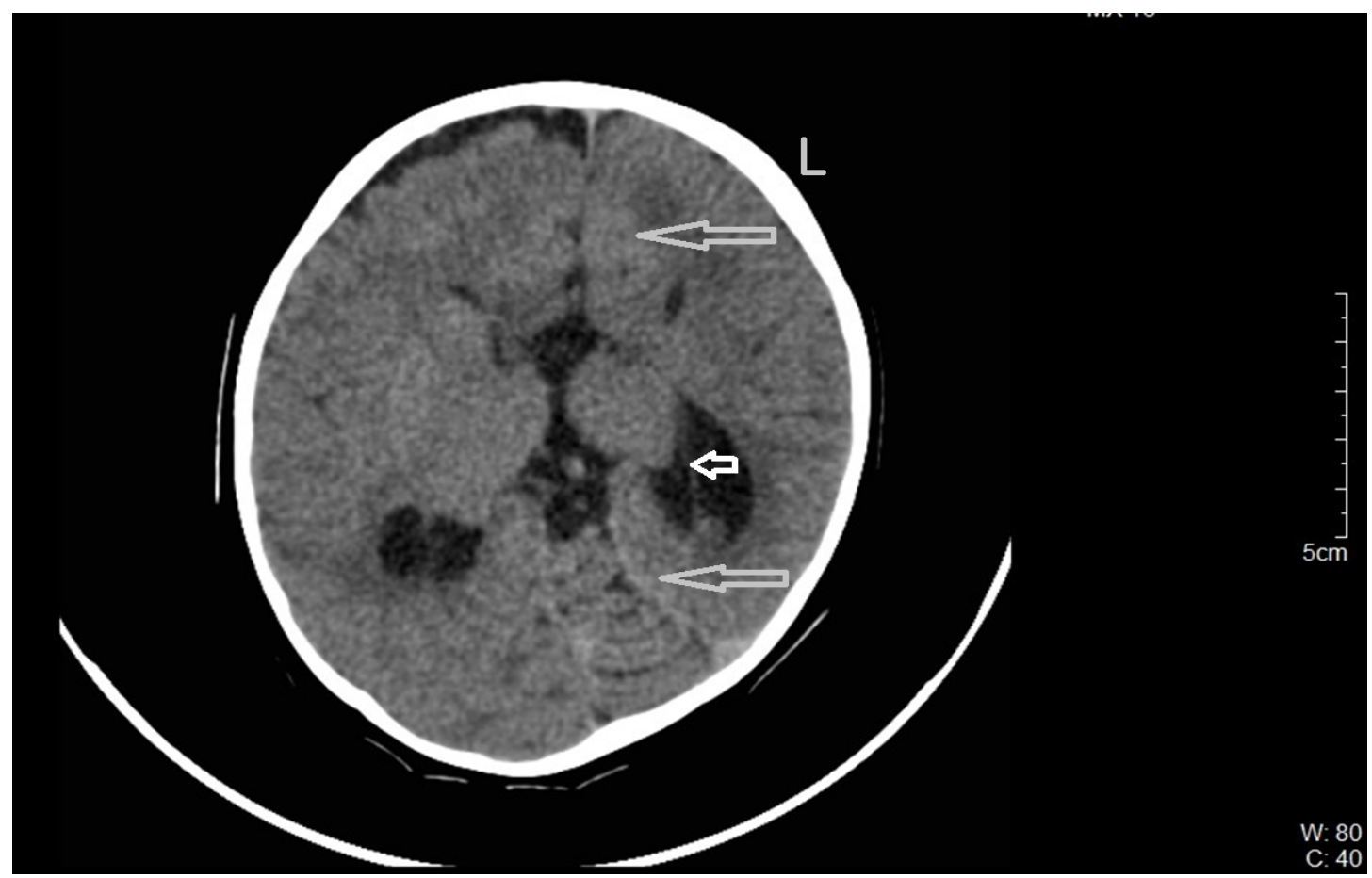

Figure 1. Computer tomography of the brain showing left cerebral atrophy (big ash arrows) and lateral ventricular dilatation (small white arrow).

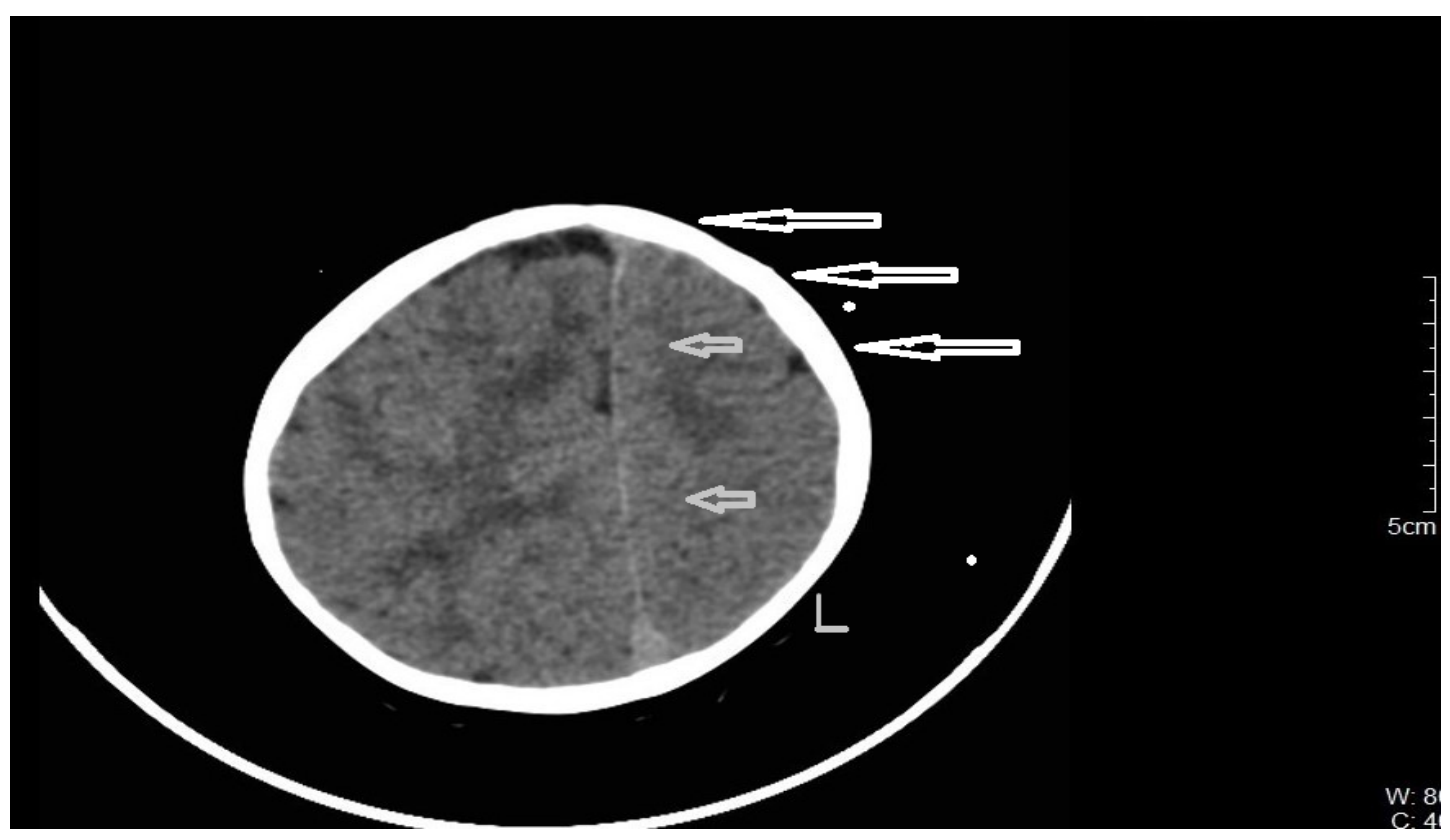

Figure 2. Computer tomography of the brain showing left cerebral atrophy (small grey arrow) and left thickened cranium (large white arrows).

Baseline complete blood count and liver function tests were normal. There was no electroencephalogram to assess the seizures. The diagnosis of cerebral palsy was initially made based 
on clinically findings. DDMS was then finally made based on the combination of the clinical findings and report of CT scan examination.

He was commenced initially on sodium valproate at a dose of $10 \mathrm{mg} / \mathrm{kg}$ per day and increased up to $40 \mathrm{mg} / \mathrm{kg}$ and later lamotrigine was added to a dose of $5 \mathrm{mg} / \mathrm{kg}$ per day. He is currently seizure free after two weeks of commencing valproate and lamotrigine and is currently on both medications. He is also receiving physiotherapy in addition. The mother said she was happy and more satisfied since the child does not have seizure and that the right limbs are becoming more functional since starting physiotherapy sessions.

\section{Discussion}

Dyke-Davidoff-Masson syndrome has been described as cerebral hemiatrophy and hypertrophy of the skull and sinuses (Zilkha, 1980). It could be seen in any age group but more in older children (Duncan et al., 2014). However, this child presented at an earlier age. There is male preponderance which was also seen in this patient (Duncan et al., 2014). It was suggested it could be congenital resulting from congenital malformation and vascular abnormalities in the middle cerebral vascular area or it could be acquired occurring intra- and post-partum from infection and birth trauma (Sharma et al., 2006). In our patient, it could be acquired occurring in the immediate postpartum period after the hypoglycaemia and hypothermia with suspected neonatal sepsis. DDMS has also been reported following cerebral malaria due to suspected cerebral occlusion (Karuppiah et al., 2009). This is not suspected in this child since malaria is highly unlikely in Lesotho and with the mother and the child having no history of travel to areas with malaria before and during pregnancy.

The clinical findings of DDMS include facial asymmetry and seizure disorders which is followed by limb weakness (Dutta et al., 2016). These presentations were all present in this patient. However, seizures occurred after the onset of weakness of the limbs. This late onset of seizures also supported the likelihood that our patient could have the acquired type as the congenital type of DDMS usually present with seizures at an earlier age.

There are some radiological features which are suggestive of DDMs. CT scan and magnetic resonance imaging (MRI) could show cerebral hemiatrophy, reduction of the white and grey matter with ipsilateral lateral ventricular dilatation, shift in midline structure to the side of cerebral atrophy and thickening of the calvarium, hyperpneumatization of the sinuses and hypoplasia of the cranial fossa (Piro et al., 2013). The CT scan of this child showed left cerebral atrophy with ipsilateral lateral ventricular dilatation. This is in line with more observation of left cerebral atrophy in other studies (Sharawat et al., 2019).

There is no definitive treatment for DDMS. The treatment is mainly symptomatic. The aim is to control seizure disorders when present as was seen in this patient. Anti-epileptic drugs have been used successfully in the management of seizures in DDMS (Malik et al, 2014). These drugs were also used in this patient. Hemispherectomy has been done with some success in some patients with intractable seizures and hemiplegia. Physiotherapy is also important for the hemiparesis management and for restoration of motor functions (Ayele \& Zewde, 2019). This was the case with our client who has clinical cerebral palsy who is currently on physiotherapy sessions. The prognosis of DDMS is better with absence of seizures and with late onset of seizures after two years of age (Duncan et al., 2014).

Conclusion: This study showed that Dyke-Davidoff-Masson syndrome still affects children despite being a condition rarely seen in health facilities especially in sub-Saharan Africa. It also showed that an appropriate assessment of clinical features and brain imaging are needed for the diagnosis of DDMS. There is need for early identification and appropriate treatment of children with Dyke-Davidoff-Mason syndrome so as to improve their daily life and general outcome. 
Informed consent and Ethics approval: Informed consent was obtained from the parent of the child. Ethical clearance was obtained from Ministry of Health Research and Ethics Committee Lesotho with reference number of Ref: ID 121-2020.

Consent to publish: A written informed consent was obtained from the patient's mother for publication of this case report. A copy of the written consent is available for review by the Editor-inChief of this journal.

Acknowledgements: We acknowledge the parent and the child with this case.

Competing interests: The authors declare no competing interest.

Authors' contributions: All authors contributed to this article. ABN contributed to the conception, design, acquisition, analysis, interpretation of data and drafting the article. CEN contributed to analysis and interpretation of data and revising it critically for important intellectual content. OFN contributed to conception and design and revising it critically for important intellectual content. MLCN contributed to conception and design and drafting the article. All authors gave the final approval for this version to be published.

\section{References}

Duncan MA, Vasguez-Flores S, Chavez-Lloevanos EB, Cantu-Salinas AC, de Leon-Flores L \& Villarreal-Velazguez HJ. (2014) Dyke-Davidoff-Masson Syndrome: a case study. Medicina Universitaria. 16: 71-73.

Dutta A, Bose S, Sen K, Pandit N \& Sharma S. (2016) Refractory seizure in childhood: DykeDavidoff-Masson syndrome revisited. Oman Med J. 31:304-308. doi:10.5001/omj.2016.58.

Karuppiah S, Christopher R \& Lombard J. (2009) Dike-Davidoff-Masson in post-cerebral malaria. J Child Neurol 24: 487-490.

Piro E, Piccione M, Marrone G, Giuffrè M, Corsello G. \& Giovanni Corsello. (2013) DykeDavidoff-Masson syndrome: case report of fetal unilateral ventriculomegaly and hypoplastic left middle cerebral artery. Ital J Pediatr 39:32 https://doi.org/10.1186/1824-7288-39-32.

Sharawat IK, Sutha R \& Sankhyan B. (2019). Dyke-Davidoff-Masson syndrome: unusual cause of hemiplegic cerebral palsy. BMJ Case Report 12:4. http://dx.doi.org/10.1136/bcr-2019-229862

Sharma S, Goyal D, Negi A, Sood RG, Jhobta A \& Surya M. (2006) Dyke-Davidoff-Masson syndrome. Indian Journal of Radiology and Imaging 16:165.

Uduma FU, Emejulu JC, Motah M, Okere PCN, Ongolo PC \& Muna W. (2013) Differential diagnoses of cerebral hemiatrophy in childhood: a review of literature with illustrative report of two cases. Glob J Health Sci 5:195-207. Doi:10.5539/gjhs.v5n3p195. https://www.ncbi.nlm.nih.gov/pmc/articles/PMC4776813/.

Zilkha A. (1980) CT of cerebral hemiatrophy. American Journal of Roentgenology. 1;135(2):259-62. 